\title{
In Vitro and In Vivo Imaging of Peptide-Encapsulated Polymer Nanoparticles for Cancer Biomarker Activated Drug Delivery
}

\author{
Gulsim K. Kulsharova*, Matthew B. Lee, Felice Cheng, Munima Haque, Hyungsoo Choi, Kyekyoon Kim, \\ William D. O’Brien, Jr, and G. Logan Liu
}

\begin{abstract}
Gelatin nanoparticles coated with Cathepsin D-specific peptides were developed as a vehicle for the targeted delivery of the cancer drug doxorubicin (DOX) to treat breast malignancy. Cathepsin D, a breast cancer cell secretion enzyme, triggered the release of DOX by digesting the protective peptide-coating layer of nanoparticles. Fabricated nanoparticles were successfully detected with ultrasound imaging in both in vitro conditions and in vivo mouse cancer models. Cell viability experiments were conducted to determine the efficacy of biomarker activation specific to breast cancer cell lines. These experimental results were compared with the outcome of a viability experiment conducted on noncancerous cells. Viability decreased in human MCF7 mammary adenocarcinoma and mouse 4T1 mammary carcinoma cells, while that of noncancerous 3T3 fibroblast cells remained unaffected. Next, a real-time video of nanoparticle flow in mouse models was obtained using in vivo ultrasound imaging. The fluorescent profile of $\mathrm{DOX}$ was used as a means to examine nanoparticle localization in vivo. Results show the distribution of nanoparticles concentrated primarily within bladder and tumor sites of subject mice bodies. These findings support the use of biomarker coated nanoparticles in target specific therapy for breast cancer treatment.
\end{abstract}

Index Terms-Chemotherapy, doxorubicin, gelatin nanoparticles, in vivo ultrasound imaging, targeted drug delivery.

\section{INTRODUCTION}

$\mathbf{T}$ HE MOST important aspect of chemotherapy is delivering high doses of drug molecules to tumor sites for maximum treatment efficacy while minimizing side effects

Manuscript received October 16, 2012; revised June 02, 2013; accepted June 21, 2013. Date of publication August 15, 2013; date of current version January 07,2014 . This work was supported in part by the University of Illinois Center for Nanoscale Science and Technology (CNST), the National Cancer Institute-funded Siteman Center for Cancer Nanotechnology Excellence at Illinois, Mesothelioma Applied Research Foundation, the Korean Ministry of Commerce, Industry and Energy under GRN Grant \#E00027, U.S. ARMY Medical Research Acquisition Activity under Contract \# W81XWh-10-1-0859 and NIH R37EB002641. Asterisk indicates corresponding author.

*G. Kulsharova is with the Department of Electrical and Computer Engineering, University of Illinois at Urbana-Champaign, IL 61801 USA (e-mail: gkulshar@gmail.com).

M. B. Lee is with the School of Integrative Biology, University of Illinois at Urbana-Champaign, IL, 61801 USA (e-mail: mblee2@uiuc.edu).

F. Cheng, M. Haque, and H. Choi are with the Department of Electrical and Computer Engineering, University of Illinois at Urbana-Champaign, IL 61801 USA (e-mail: fcheng3@gmail.com; haque2@illinois.edu; hyungsoo@illinois. edu).

K. Kim, W. D. O'Brien, and G. L. Liu are with the Department of Electrical \& Computer Engineering and with the Department of Bioengineering, University of Illinois at Urbana-Champaign, IL 61801 USA (e-mail: wdo@uiuc.edu; loganliu@illinois.edu; kevinkim@illinois.edu).

Color versions of one or more of the figures in this paper are available online at http://ieeexplore.ieee.org.

Digital Object Identifier 10.1109/TNB.2013.2274781 to normal organs and tissues. Off-target chemotherapeutic uptake by healthy tissues often causes systemic damages and compromised treatment efficiency. For example, one of the most effective and widely used anticancer drugs, doxorubicin (DOX), is reported to cause serious adverse effects including nausea, vomiting, anorexia and most adversely, heart damage (cardiotoxicity), which considerably limits its applicability [1]-[3]. Therefore, the prevention of systemic drug release is crucial to improving chemotherapy.

Of several strategies that have been proposed to target cancer cells, those based on biodegradable and biocompatible polymeric particles have attracted considerable attention due to their stability [4], versatility of surface modification [5], and potential for different drug release characteristics [6]-[8]. Drug-carrying particles or macromolecular drugs are reported to accumulate more selectively at or near the tumor sites due to the enhanced permeability and retention (EPR) effect of tumor vasculature, mitigating off-target drug delivery [9]-[11]. Although the efficiency of drug delivery increases with molecular or nanoparticle drug carriers, off-target drug delivery or release still exists due to the fast drug release from nanoparticles during circulation. This calls for a stable carrier of potent cancer therapeutics that releases the drug only at the site of the tumor.

One targeting strategy deriving from the need for a drug release to occur near the tumor may be to conjugate particle surfaces with antibodies that can detect tumor-associated antigens, which was reported to be successful primarily for in vitro screening [12], [13]. Despite these promising in vitro results, in vivo application might be restricted due to the weak linkage stability, potential immunogenicity after repeated injections [14] and genetic diversification of tumor cells. A better strategy might be to design a peptide specific to the over-expressed secretory proteins at cancer sites which have shown to be promising biomarkers for in vivo diagnostics or prognostics of tumors [15] due to their higher stability and less potent immunogenicity. Among these proteins are proteases and peptidases which catalyze the hydrolysis of peptide bonds with high specificity [16] and, therefore, may be used to activate drug release thus serving as a new tool for cancer targeting.

Additionally, for effective nanoparticle-based systems, it is essential to monitor drug delivery to targeted sites and verify the efficacy of the encapsulating peptide/antibody layer. However, it is difficult to monitor drug carrier transport due to lack of sufficient contrast in current monitoring systems. Systemic drug release can be prevented and specificity can be achieved by engineering the drug nanocarriers to improve current chemotherapy. 


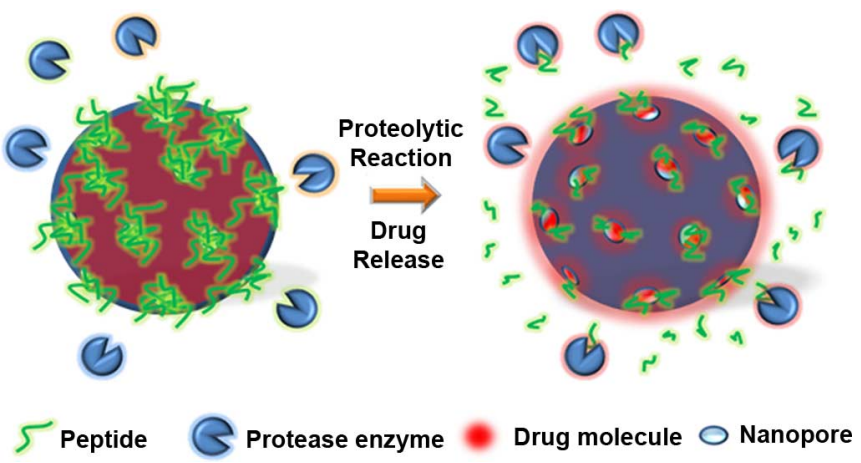

Fig. 1. Illustration of gelatin nanoparticle drug carrier protected by protease substrate peptides. Left side of the figure shows the drug-loaded nanoparticles coated with peptide strands before the nanoparticles are affected by enzyme, which is shown as blue particles surrounding nanoparticles. The right side of the figure is illustration of peptide cleavage and drug release that occurs after proteolytic reaction takes place.

We report a novel gelatin nanoparticle carrier for the targeted delivery of DOX to treat breast malignancies, which avoids problems of early nonspecific dissolution and off-target drug release and is suitable for high-resolution ultrasound and fluorescence imaging in animal models. The versatile nanotechnology here could be applied to treatment of different kinds of cancer with the change of biomarker specific peptides. A schematic diagram of the chemotherapeutic drug delivery vehicle is shown in Fig. 1. The nanoparticle core was fabricated by the Electric Field Assisted Precision Particle Fabrication (E-PPF) method using acidic gelatin, loaded with DOX [17], [18]. The resulting nanospheres were coated with a high-density peptide layer, the hydrolysis of which is catalyzed by Cathepsin $\mathrm{D}$, a specific biomarker protease hypersecreted by breast cancer cells. Thus, the core is protected from general proteolysis, wherein DOX is safely contained, until the digestion of the peptide shell is catalyzed by the secretory protease enzyme Cathepsin D in the proximity of breast cancer cells. As the peptide shield is removed, gelatin is exposed to general proteases abundant in all cell secretions, triggering the release of DOX. As a result, the drug is released only in the vicinity of the target cancer cells and its release dosage is controlled by the localized secretory protease concentration. Due to the low concentration of targeted protease in benign tissues, the peptides covering the nanocapsule surface remain intact and the drug inside the nanocapsule is well contained. By this, most effective chemotherapy may be achieved with minimal side effects.

\section{METHODS}

\section{A. Fabrications and Characterization of Drug Nanoparticles}

Gelatin (225 g bloom, BioReagent) nanoparticles were fabricated by an E-PPF method [18] and were cross-linked using $200 \mathrm{~mL}$ of $0.125,0.375,0.625$, and $0.875 \% \mathrm{w} / \mathrm{v}$ glutaraldehyde (GA) (25\% aqueous solution, Sigma-Aldrich) solutions at $4{ }^{\circ} \mathrm{C}$ for 24 hours, followed by the addition of glycine (Sigma-Aldrich) at room temperature to deactivate the remaining GA [17]. The resulting nanoparticles were washed with DI water and lyophilized. Morphology and uniformity of particles were studied by scanning electron microscopy (SEM, Hitachi S-4700). DOX molecules (Sigma-Aldrich) were loaded into the gelatin matrix through impregnation using $1 \mathrm{mg} / \mathrm{mL}$ drug solutions. Upon inward diffusion the drug molecules were ionically bound to the gelatin matrix, preventing their release in the absence of enzyme.

In order to keep the drug inside the capsule, the peptide strands were synthesized, which were then conjugated to the surface of the gelatin nanoparticles. $500 \mu \mathrm{L}$ of $0.1 \mathrm{M}$ PBS solution, $100 \mu \mathrm{L}$ of $0.33 \mathrm{mM}$ 1-ethyl-3-(3-dimethylaminopropyl) carbodiimide hydrochloride (EDC) (Sigma-Aldrich), and $100 \mu \mathrm{L}$ of $0.5 \mathrm{mM}$ N-hydroxysuccinimide (NHS) (Sigma-Aldrich) solution were added to make EDC-NHS PBS solution as catalyst for the conjugation [19]. The peptides here are attached through covalent bonding between the crosslinking amine moiety groups at the peptide terminal and the carboxyl groups on the surface of the gelatin nanoparticles, cross-linked via GA.

The peptide for targeting contains a Phe-Phe-Arg-Asp sequence, a blue fluorophore molecule at the $\mathrm{C}$-terminus, and a darker quencher molecule near the $\mathrm{N}$-terminus (synthesized by BioMol). To the prepared mixture, $0.2 \mathrm{~mL}$ of $0.1 \mu \mathrm{M}$ peptide and drug-loaded gelatin nanoparticles were added and kept overnight. The resulting mixture was centrifuged and washed with dimethylsufoxide to collect peptide-conjugated particles. Drug-loaded particles were incubated with purified Cathepsin D and culture media of MCF7, 3T3, and HeLa cells, and fluorescence intensity of the surface peptides (excitation of 328 $\mathrm{nm}$ and emission of $393 \mathrm{~nm}$ ) was measured at designated time intervals using a microplate reader (BioTek Synergy).

The specificity of the designed peptide substrate to the Cathepsin D secreted by breast cancer cells was examined via the fluorescence emission from the terminal methoxycoumarin (MCA) fluorophore molecule. The fluorescence was quenched by the dinitrophenyl (Dnp) molecule before the proteolytic reaction due to near field fluorescence energy transfer between the MCA fluorophore and Dnp quencher molecule.

\section{B. In Vitro Chemotherapy}

To evaluate targeted cell specificity, nanoparticle mediated chemotherapy on three different types of cells was carried out. MCF7 human mammary adenocarcinoma, 4T1 mouse mammary carcinoma, and 3T3 mouse fibroblast were chosen (all from ATCC, Manassas, VA) for the experiment. ATCC-formulated Eagle's Minimum Essential Medium with $0.01 \mathrm{mg} / \mathrm{ml}$ bovine insulin, $10 \%$ fetal bovine serum (FBS) was used as a culture medium for MCF7, while 3T3 Swiss mouse fibroblast cells (ATCC) were cultured using ATCC-formulated Dulbecco's Modified Eagle's Medium mixed with bovine calf serum to a final concentration of $10 \%$. 4T1 mouse breast cancer cells were cultured using RPMI-1640 media mixed with $10 \%$ FBS. All media were filtered using a $0.22 \mu \mathrm{m}$ vacuum filter for sterilization. The cells were added to the cultured media and then kept in 75 sq. cm flasks for culturing in incubator 5\% carbon dioxide at $37^{\circ} \mathrm{C}$.

In addition to the mentioned cell cultures, HeLa cancer cells were also cultured for the purpose of using them in peptide hydrolysis and DOX drug release studies. To evaluate the drug release profile, study on in vitro release of DOX from the drugloaded carriers with and without peptide surface coating was performed in the presence of $0.01756 \mathrm{mg} / \mathrm{mL}$ Cathepsin D enzyme, MCF7 culture media, 3T3 culture media, HeLa culture 
media, and $9.9 \mathrm{mg} / \mathrm{mL}$ collagenase $1 \mathrm{~A}$, respectively. Drug release was assessed by sampling the supernatant and measuring the fluorescence emission from the DOX molecules using a microplate reader (excitation of $470 \mathrm{~nm}$ and emission of $585 \mathrm{~nm}$ ). Protease-, DNAs-, and RNAs- free water (Fisher Scientific) was used for the measurement.

To evaluate the chemotherapy on cancer cells, MCF7, 4T1 and $3 \mathrm{~T} 3$ cell cultures were incubated for $7 \mathrm{~h}$ with drug loaded and peptide coated $2 \times 10^{6}$ nanoparticles mixed in $0.1 \mathrm{~mL}$ of PBS solution. Optical images were taken every 2 to $3 \mathrm{~h}$ and cell viability was measured at different time points.

Additionally, a MTT cell proliferation assay (ATCC) was carried out for human breast MCF7 and mouse breast 4T1 cancer cells. This assay provides a reliable and quantifiable means of measuring the absorbance values of cell populations through spectrophotometry. Each 30000 cells $/ \mathrm{mL}$ solution of $4 \mathrm{~T} 1$ cells and 38000 cells $/ \mathrm{mL}$ solution of MCF7 cells were placed in 2 and 4 wells of 96-well plates in equal amounts for each cell line, respectively. While half of the wells for each cell line were treated with drug carrying nanoparticles, the remaining half was incubated without nanoparticles for control measurements. Cell concentrations of treated MCF7 and 4T1 cells were assessed after incubation time using the MTT assay for viability and compared to those in control wells.

\section{In Vivo Chemotherapy}

For this stage of the study, the experimental protocol was approved by the Institutional Animal Care and Use Committee of the University of Illinois and satisfied all campus and National Institutes of Health rules for the humane use of laboratory animals. Animals were housed in an Association for Assessment and Accreditation of Laboratory Animal Care (Rockville, MD)-approved animal facility and provided food and water $a d$ libitum.

In vivo evaluation of proposed nanoparticles was carried out on nude mice models. For in vivo ultrasound detection nude mice (Harlan, Indianapolis, IN) weighing 19-21 g were injected via the lateral tail vein with $100 \mu \mathrm{L}$ of saline solution containing $2 \times 10^{9}$ nanoparticles per $\mathrm{mL}$ that ranged between 200 and 900 $\mathrm{nm}$ in diameter. Real-time video of nanoparticle flow in the superior vena cava was captured starting from the injection time using a Vevo 2100 High Frequency Ultrasound Imaging System (VisualSonics, Toronto), indicating the potential application of the drug carrying nanoparticles in serving as ultrasound imaging contrast agents.

For tumor induction, nude mice were anesthetized under isofluorane ( $2 \%$ isofluorane, $2 \%$ oxygen flow rate) and subcutaneously injected with $100 \mu \mathrm{L}$ of media containing $1 \times 10^{5}$ 4T1 cells in the lower abdominal mammary gland. Tumors were allowed to grow to a maximum diameter of $10 \mathrm{~mm}$ before nanoparticle exposure. These 4T1 tumor mice were then injected with $100 \mu \mathrm{L}$ of saline solution containing $2 \times 10^{8}$ peptide-coated drug nanocarriers per $\mathrm{mL}$. Due to the naturally fluorescing nature of DOX, fluorescence images of the injected and control mice were obtained for accessing the distribution of the drug in the mouse body after injection. Based on the fluorescent profile of DOX, an excitation of $470 \mathrm{~nm}$ and emission of $590 \mathrm{~nm}$ was used.

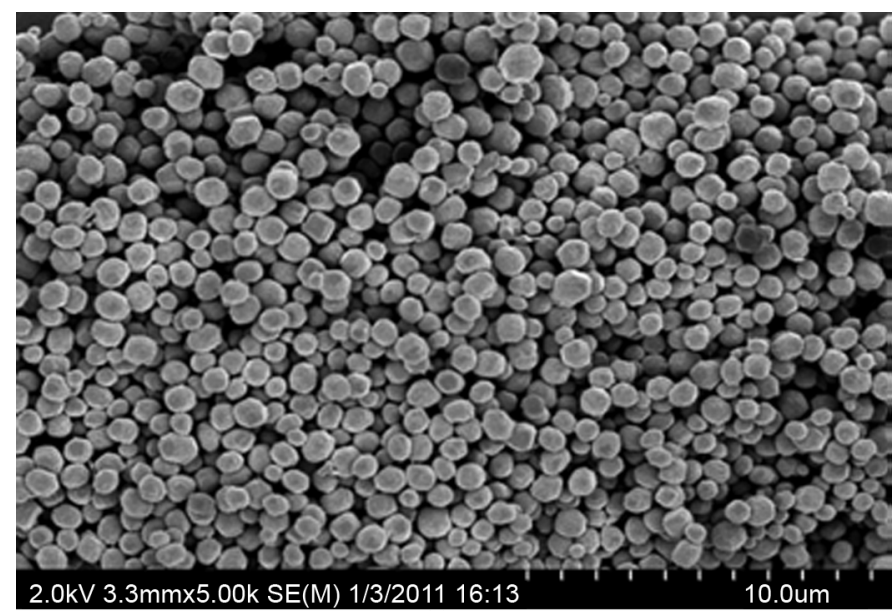

(a)

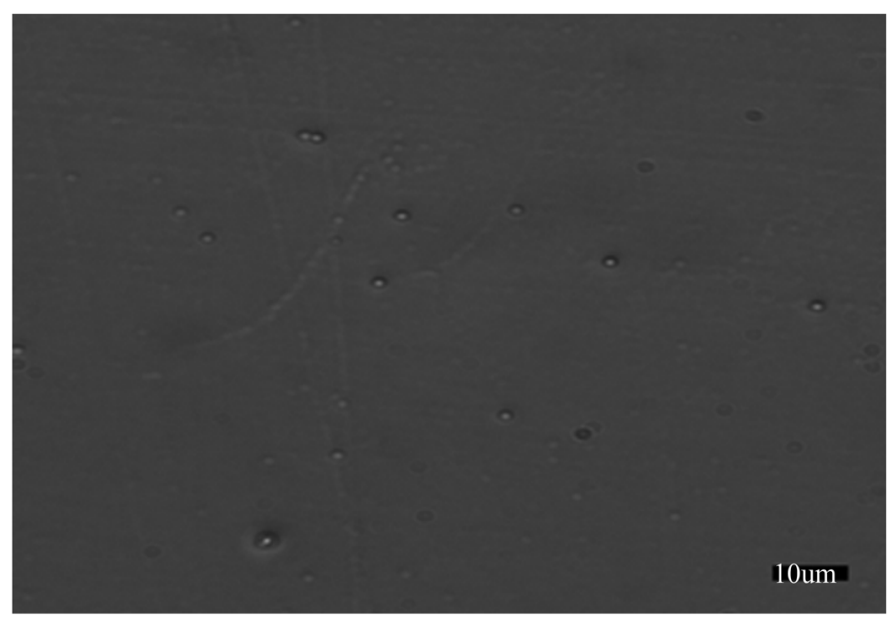

(b)

Fig. 2. Gelatin nanoparticle drug carriers. (A) Scanning electron microscope images of gelatin submicron particles (mean diameter of $1 \mu \mathrm{m}$ ); (B) Optical image of gelatin particles after swelling in saline solution. $(200 \mathrm{~nm}-5 \mu \mathrm{m})$.

\section{RESUlTS AND Discussions}

\section{A. Gelatin Nanoparticles}

The scanning electron microscope (SEM) image in Fig. 2(a) and optical image of particles in saline solution (Fig. 2) show that the particles fabricated by the E-PPF method are spherical and uniform in size. The swelling ratio, defined as the ratio of diameters of wet $(1.62 \mu \mathrm{m})$ and dry $(0.9 \mu \mathrm{m})$ particles, was 1.8 .

The specificity of the designed peptide to Cathepsin D was examined via fluorescence emission from the terminal MCA fluorophore molecule on the peptide strand, which is quenched by the Dnp molecule before the proteolytic reaction due to near field fluorescence energy transfer. Fig. 3(a) schematically illustrates gelatin particles conjugated with peptides containing a Leu-Phe-Phe-Arg-Leu sequence, which can be recognized by Cathepsin D, an aspartic protease enzyme prominent in breast malignancies [12]. Once hydrolysis of the peptide is catalyzed by Cathepsin D, the peptide substrate fluoresces as an indicator of the proteolytic activity of the peptide coating layer on the nanoparticle surface. When the peptide-coated particle loaded with DOX is incubated respectively, with purified Cathepsin D and with the MCF7 breast cancer cell media, the fluorescence 


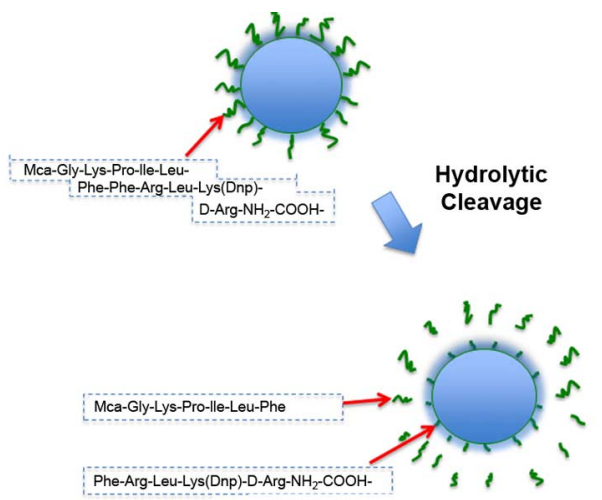

(a)

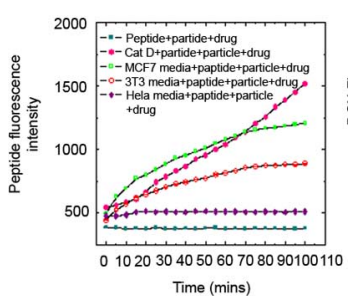

(b)
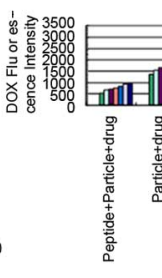

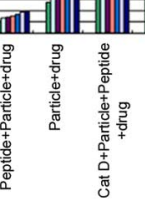

Fig. 3. Peptide hydrolysis and DOX drug release from the gelatin particles (A) Schematic illustration of peptide-conjugated nanoparticles. The peptide sequence is shown. The peptide can be hydrolyzed at Phe-Phe bond by Cathepsin D enzymes and then show fluorescence. (B) Peptide fluorescence intensity under different incubated conditions. (C) Fluorescence intensity of released DOX drug under various conditions. The tested samples include pure buffer solution, purified Cathepsin D solution, purified Collagenase 1A solution, and 2-day-incubation culture media of MCF7 human breast cancer cells, $3 \mathrm{~T} 3$ mouse fibroblast cells, and HeLa human cervical cancer cells.

intensity, as shown in Fig. 3(b), increases. This observation indicates active proteolytic reactions on the particle surface. On the other hand, the blue fluorescence intensity remains unchanged when the particles are incubated with nontargeted protease enzymes, e.g., collagenase $1 \mathrm{~A}$ and nontargeted human cell lines (e.g., HeLa cells), which strongly indicates the specificity of the peptide layer to the targeted cancer biomarker, in this case Cathepsin D. For 3T3 mouse fibroblast cell secretions, possibly due to the nonspecific proteolytic reactions of the peptides, the peptide fluorescence intensity is also elevated but the elevation level and sustainability are significantly lower than those for MCF7 mammary adenocarcinoma cells. Better design of peptides with higher specificity also will help to minimize the nonspecific proteolytic reactions.

\section{B. In Vitro Release Profile of DOX-Loaded Particles}

The release profiles of DOX from particles with and without the peptide coating incubated in pure buffer solution, purified Cathepsin D solution, purified collagenase 1A solution, secretion with culture media of MCF7 human breast cancer cells, 3T3 mouse fibroblast cells and HeLa cells, respectively, were expressed as DOX fluorescence intensity [Fig. 3(c)] which is assumed to be proportional to the drug concentration. In comparison to peptide-coated nanoparticles, increased DOX release was seen in uncoated nanoparticles incubated in conditions with and without collagenase, a common protease that facilitates the hydrolysis of gelatin. This observation suggests that the peptide coating layer hinders the diffusive drug release and degradation of gelatin catalyzed by collagenase. The release of DOX from the peptide-coated particles was prohibited in most of the cases except for the purified Cathepsin D solution and MCF7 breast cancer cell secretion. The drug release variation between the above two cases might be attributed to the different protease concentration leading to different degradation rate or other proteases secreted by MCF7 cells, such as collagenase. In sharp contrast, the uncoated nanoparticle drug carrier had considerable natural diffusion-driven drug release and biodegradation even when it was not incubated with cancer cells. Due to nonspecific proteolytic reactions in the case of $3 \mathrm{~T} 3$ mouse fibroblast cell secretion, minor DOX release was observed but the amount was significantly lower than that in MCF7 human breast cancer cell secretions. The enhancement of DOX release was also observed in both coated and uncoated nanoparticles cultured with collagenase, which is ascribable to collagenase-assisted hydrolysis of gelatin leading to the dissociation of the drug carriers. Drug release, however, is still much suppressed compared to the bare nanoparticles with or without the presence of collagenase. This evidence once again supports that the peptide layer on the particle surface dramatically diminishes the nonspecific drug release and improves specificity to the targeted cancer biomarkers.

\section{Targeted Chemotherapy on Breast Cancer Cells}

The morphology of peptide-coated nanoparticles cultured with three different cell lines was examined along with the number of resulting viable cells. MCF7 mammary adenocarcinoma cells and 4T1 mouse mammary carcinoma cells were specifically chosen to show that the peptide specificity to Cathepsin D secreted by these cells, while $3 \mathrm{~T} 3$ mouse fibroblasts were used as controls. Nanoparticles incubated with 4T1 cells significantly reduced the cell growth in the span of $7 \mathrm{~h}$ [Fig. 4(a)], but did not affect the growth rate of 3T3 cells [Fig. 4(a)]. The number of 3T3 fibroblasts increased due to cell proliferation, which indicates negligible cytotoxicity to these nontargeted cells. In contrast, the number of 4T1 cells decreased dramatically by more than $80 \%$ after $5 \mathrm{~h}$ and kept this downward trend temporally [Fig. 4(a)]. The number of MCF7 cells, although with some oscillation temporally, eventually decreased to $50 \%$ of its initial concentration after $7 \mathrm{~h}$. These results demonstrate that the peptide coating enables specificity to only target cancer biomarkers and associated tumor cells. Results of these experiments using mice and human breast cancer cells are shown in Figs. 4(b) and 4(c). Cell viability of treated and untreated MCF7 and 4T1 cells were assessed after a 2-h incubation time for each cell line. The data strongly suggest that cell viability was significantly reduced for cells incubated with nanoparticles, while control cells that were untreated with nanoparticles continued their growth, reinforcing the same result obtained in the discussed Fig. 4(a). While the concentration of $4 \mathrm{~T} 1$ cells decreased from 30000 to 26000 cells $/ \mathrm{mL}$ by the end of incubation time, the concentration of control 4T1 cells remained unaffected by nanoparticles, increasing to 34000 cells $/ \mathrm{mL}$. Analogous trends for MCF7 cell growth were obtained. Thus, results demonstrate significant effects of drug-loaded nanoparticles conjugated with peptides on breast cancer cell viability. 


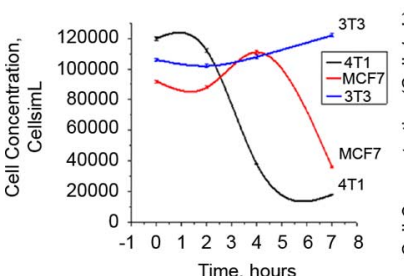

(a)

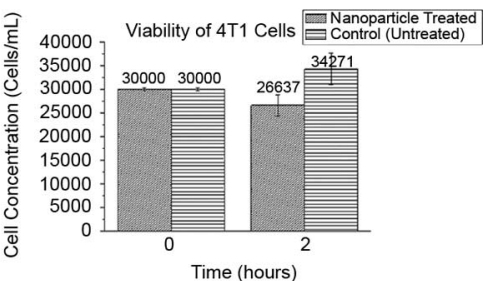

(b)

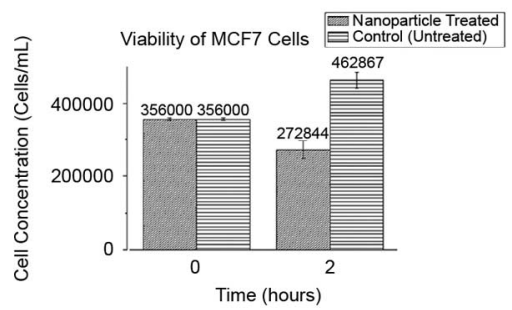

(c)

Fig. 4. In vitro cancer cell chemotherapy using peptide-conjugated gelatin nanoparticles as DOX drug carrier. (A) Cell counting for 3T3 (control) and 4T1 cells in 7 hour duration. 3T3 viability curve suggest that this noncancerous cell line is not affected by nanoparticle incubation. The viability of $3 \mathrm{~T} 3$ cells treated with nanoparticles increases, unlike 4T1 cell viability, which significantly decrease after incubation with nanoparticles. (B) Comparison of number of viable cells for 4T1 cells treated and untreated with fabricated nanopartciles. (C) Comparison of number of viable cells for MCF7 cells treated and untreated with nanopartciles. Figs. 4(b) and 4(c) show that viability of nanoparticle treated human and animal breast cancer cells decrease unlike untreated control cells. Fig. 4(a) shows that viability of 3T3 is not affected by fabricated nanoparticles and cell viability of $3 \mathrm{~T} 3$ cells treated with nanoparticles increases unlike mouse cancer 4T1 cell viability that significantly decreases after incubation with nanoparticles.

\section{In Vivo Ultrasound Imaging of Nanoparticles}

Gelatin nanoparticles were injected into control mice via the lateral tail vein and real-time video of the superior vena cava was taken immediately after the injection. Snapshots of the particles passing through the vein located near the mouse heart are shown in Figs. 5(a) and 5(b). Fig. 5(a) shows the vena cava before introducing the particles into the body, while Fig. 5(b) shows gelatin particles passing through the vein. Results suggest that the proposed gelatin nanoparticles can provide sufficient contrast to facilitate in vivo high-resolution ultrasound imaging. This observation could be due to swelling characteristic of the nanoparticles, which causes the formation of air gaps and free pores, giving them distinctive acoustic impedance. As a result, the particles can act as reflective mediums for ultrasound waves, allowing in vivo ultrasound detection, tracking of particle flow, and distribution in real time.

\section{E. Targeted Chemotherapy on Breast Cancer Mice Models}

The fluorescence nature of DOX allowed us to use imaging techniques for monitoring the distribution of nanoparticles in vivo. Fluorescence imaging was performed to demonstrate peptide coating stability and nanoparticle drug release specificity to biomarkers secreted by tumor sites under in vivo conditions. As previously described both mice with and without cancerous growths were injected with $100 \mu \mathrm{L}$ of fabricated particles mixed in saline solution and underwent whole body fluorescence imaging using a small animal fluorescence imaging system. The fluorescence images are overlaid on the bright field images to identify the locations of nanocapsules present in the mice (Fig. 6). The middle image in Fig. 6 shows that most

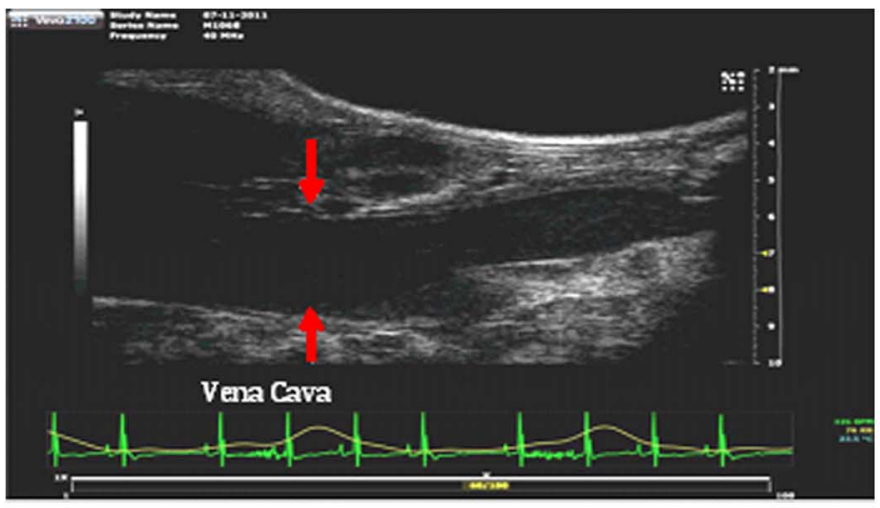

(a)

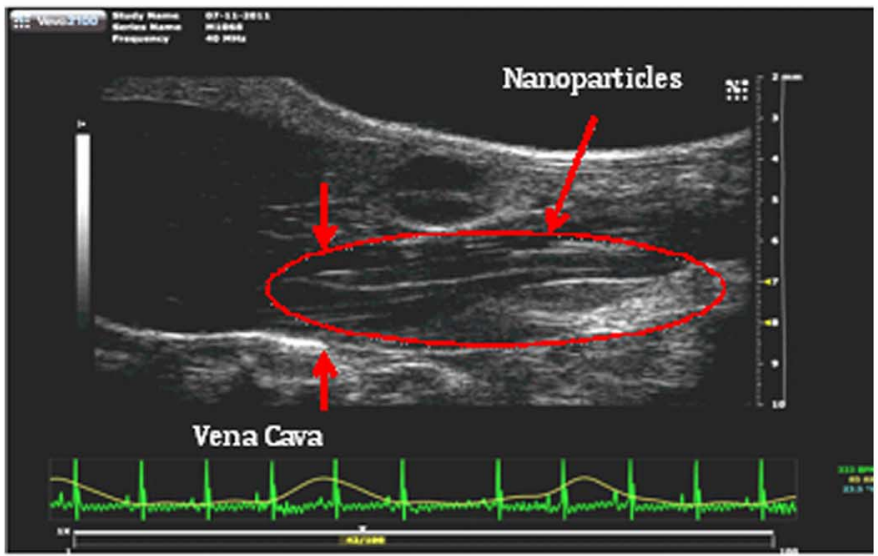

(b)

Fig. 5. High frequency ultrasound images of the blood vessel in the heart of a nude mouse during the injection of nanocapsules. Ultrasound imaging of vena cava vessel (A) before and (B) several seconds after the injection of particles via the tail vein into the mouse body. The nanocapsules in flow can be clearly identified on Fig. 5(b) circled with red inside the vena cava.

of the particles are filtered out and end up in the bladder, while the image of the 4T1-tumor shows that proposed nanoparticles concentrate not only in the bladder, but also at the tumor site. Evidently, DOX fluorescence is strong in the tumor and at the locations near the tumor, suggesting the targeted delivery through the nanocapsules. Thus, the concentrated distribution of drug carrying nanoparticles in the 4T1 tumor supports the hypothesis that introduced particles are biomarker activated through peptide encapsulation.

\section{CONCLUSION}

\section{A. Conclusion, Limitations and Future Perspectives}

In this study, nanoporous gelatin nanoparticles were fabricated as a vehicle for high specificity and targeted drug delivery to breast cancer cells. Chemotherapeutic DOX drug molecules were loaded in gelatin nanocarriers and coated with protective peptide strands to prevent early degradation and nonspecific drug release. Release of drug immobilized by cross-linked gelatin, the loading efficiency of which can be optimized by controlling the cross-linker concentration, is triggered by nothing but the biomarker protease enzyme Cathepsin D. In comparison with the chemotherapy by naked drugs or encapsulated drugs without a protective shell, the peptide-coated nanospheres can significantly improve the specificity of cancer 


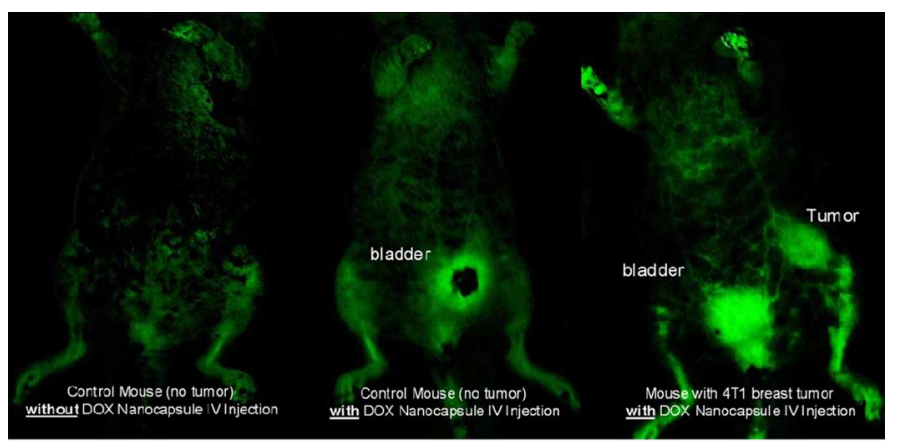

(a)

(b)

(c)

Fig. 6. Fluorescence imaging studies on cancerous mice models. (A) Fluorescence image of control cancer free mouse not injected with DOX nanocapsules; (B) Fluorescence image of control cancer free mouse model after the injection of drug loaded nanoparticles coated with peptide layer; (C) Fluorescence image of a cancerous mouse model with 4T1 breast tumor after injection of nanoparticles. Green color is a pseudocolor corresponding to DOX fluorescence intensity. Since fluorescence intensity from peptide (excitation of $328 \mathrm{~nm}$ and emission of $393 \mathrm{~nm}$ ) is different that the fluorescence profile for DOX drug (an excitation of $470 \mathrm{~nm}$ and emission of $590 \mathrm{~nm}$ ), it was filtered out in these imaging studies and it does not contribute to the fluorescence shown on the images.

chemotherapeutic drug delivery and mitigate the adverse side effects due to off-target drug release. Varying the peptide sequences for surface coating can also target different cancer biomarkers, the nature of which depends on the cancer type. Thus, hybrid drug carriers may be tailored for different subtypes of cancers for personalized medicine and therapy.

The nanoscale size of particles allowed us to extend the studies to in vivo drug delivery. We achieved sufficient contrast for fabricated gelatin nanocarriers during in vivo ultrasound imaging to monitor the nanocarrier transport after its IV injection. The targeted drug delivery scheme was successfully demonstrated in vitro and in vivo for breast cancer malignancy with fluorescence imaging techniques. We anticipate that with higher specificity and stability of our peptide conjugated drug nanocarriers, systemic drug release and off-target drug delivery problems can be addressed to remove adverse side effects of current cancer malignancy treatments.

Along with encouraging results, this study poses some limitations, however, and more work will be needed to fully demonstrate the efficacy of the nanoparticles. Due to the complexity of experiments and time required for preliminary preparation of animal models, we were only able to conduct fluorescence imaging experiments on one set of animals. These experiments could be carried out with a larger animal sample in order to better ensure the reliability of the results.

Secondly, drug-carrying particles and macromolecular drugs are reported to accumulate by tumor sites due to the EPR (enhanced permeability and retention) effect. The EPR effect might have contributed to the intensity of released DOX by tumor sites in our fluorescence imaging experiments. In order to determine how much of this DOX intensity is caused by the EPR effect as well as how much is caused by peptide cleavage and DOX release, more experiments involving cancerous mice models are suggested. A cancerous mouse model injected with our nanoparticles could be compared with a cancerous model injected with drug-loaded nanoparticles without peptide coating. Furthermore, additional in vivo fluorescence imaging can allow us to learn more about the effectiveness of drug release on tumor size. Conducting the earlier described in vivo fluorescence experiments with the purpose of monitoring the size of a tumor can be a step towards reinforcing the obtained results.

Overall, in spite of its limitations, the presented research could be a substantial contribution towards the growing development of smart nanoparticle drug carriers. Targeted drug delivery and chemotherapy has been an extremely active research area. Most of the current strategies using antibody-conjugated nanoparticle drug carrier or chemical compounds improve the therapeutic specificity to some extent. However, these nanoparticles are not "smart" enough to tune the drug release dosage, identify metastatic and circulating tumor cells or penetrate into encapsulated tumor nodules. In the presented research, however, the drug delivery dosage is fine-tuned by the localized biomarker concentration and the chemotherapy drug is released in the vicinity of the tumor rather than upon the physical binding within the tumor, resulting in potentially better penetration to deep tumors. All these unique properties make the reported enzymatically activated nanoparticle drug carrier a better one than antibody-based carriers by providing lower systemic release and high delivery efficiency. The targeted drug delivery in the coming decade will rely on nanoparticle drug carriers that actively search for potent cancer cells, diagnose the cell condition and decide drug delivery dosage and rate autonomously. After drug delivery, the nanoparticle drug carrier can dissolve itself and be cleared out of the body. Negative side effects can be reduced to nearly zero and the precision drug delivery can result in optimized, rapid and effective cancer chemotherapy.

\section{ACKNOWLEDGMENT}

In vivo whole body imaging studies were carried out in cooperation with S. Boppart, M.D. Ph.D, and E. Chaney from the Biophotonics Imaging Laboratory at the University of Illinois. The authors would also like to thank R. Abuhabsah, E. Hartman, RDMS, and R. J. Miller D.V.M for their respective assistance in tumor inducement preparation, in vivo mouse ultrasound imaging, and supervision in all animal work.

\section{REFERENCES}

[1] H. T. Ta, C. R. Dass, I. Larson, P. F. M. Choong, and D. E. Dunstan, "A chitosan-dipotassium orthophosphate hydrogel for the delivery of doxorubicin in the treatment of osteosarcoma," Biomaterials, vol. 30, no. 21, pp. 3605-3613, Jul. 2009.

[2] L. J. Wood, L. M. Nail, A. Gilster, K. A. Winters, and C. R. Elsea, "Cancer chemotherapy-related symptoms: evidence to suggest a role for proinflammatory cytokines," Oncol. Nurs. Forum, vol. 33, no. 3, pp. 535-542, May 2006.

[3] O. Tacar, P. Sriamornsak, and C. R. Dass, "Doxorubicin: an update on anticancer molecular action, toxicity and novel drug delivery systems," J. Pharmacy Pharmacol., vol. 10, Aug. 2012.

[4] R. Herrero-Vanrell, A. C. Rincón, M. Alonso, V. Reboto, I. T. MolinaMartinez, and J. C. Rodríguez-Cabello, "Self-assembled particles of an elastin-like polymer as vehicles for controlled drug release," J. Control. Rel., vol. 102, no. 1, pp. 113-122, Jan. 2005.

[5] C. Vauthier, C. Dubernet, C. Chauvierre, I. Bigger, and P. Couvreur, "Drug delivery to resistant tumors: the potential of poly (alkyl cyanoacrylate) nanoparticles," J. Control. Rel., vol. 93, no. 2, pp. 151-160, Dec. 2003.

[6] G. G. Pitt, M. M. Gratzel, G. L. Kimmel, J. Surles, and A. Sohindler, "Aliphatic polyesters II. The degradation of poly (DL-lactide), poly (å-caprolactone), and their copolymers in vivo," Biomaterials, vol. 2, no. 4, pp. 215-220, Oct. 1981.

[7] M. Barratt, "Therapeutic applications of colloidal drug carriers," Pharmaceut. Sci. Tech. Today, vol. 3, no. 5, pp. 163-171, May 2000. 
[8] P. Couvreur, C. Dubernet, and F. Pusieux, "Controlled drug delivery with nanoparticles: current possibilities and future trends," Eur. J. Pharm. Biopharm., vol. 41, no. 1, pp. 2-13, 1995.

[9] H. Maeda, J. Wu, T. Sawa, Y. Matsumura, and K. Hori, "Tumor vascular permeability and the EPR effect in macromolecular therapeutics: a review," J. Control. Rel., vol. 65, no. 1-2, pp. 271-284, 2000.

[10] H. Maeda, "The enhanced permeability and retention (EPR) effect in tumor vasculature: the key role of tumor-selective macromolecular drug targeting," Adv. Enzyme Regul., vol. 41, no. 41, pp. 189-207, 2001.

[11] A. K. Iyer, G. Khaled, J. Fang, and H. Maeda, "Exploiting the enhanced permeability and retention effect for tumor targeting," Drug Discov. Today, vol. 11, no. 17-18, pp. 812-818, Sep. 2006.

[12] N. Ferrara, K. J. Hillan, H. P. Gerber, and W. Novotny, "Discovery and development of bevacizumab, an anti-VEGF antibody for treating cancer," Nat. Rev. Drug Discov., vol. 3, pp. 391-400, May 2004.

[13] M. Arruebo, M. Valladares, and A. Gonzalez-Fernandez, "Antibodyconjugated nanoparticles for biomedical applications," J. Nanomater., vol. 2009, pp. 24-24, Sep. 2009.

[14] P. A. Trail, D. H. King, and G. A. Dubowchik, "Monoclonal antibody drug immunoconjugates for targeted treatment of cancer," Cancer Immunol. Immunother., vol. 52, no. 5, pp. 328-337, Jan. 2003.

[15] M. E. Akerman, W. C. W. Chan, P. Laakkonen, S. N. Bhatia, and E. Ruoslahti, "Nanocrystal targeting in vivo," Proc. Natl. Acad. Sci. USA, vol. 99, p. 12617, 2002.

[16] N. D. Rawlings, F. R. Morton, and A. J. Barrett, "MEROPS: the peptidase database," Nucleic Acids Res., vol. 27, no. 1, pp. 325-331, Jan. 1999.

[17] Y. B. Choy, F. Cheng, H. C. Choi, and K. Kim, "Monodisperse gelatin microspheres as a drug delivery vehicle: Release profile and effect of crosslinking density," Macromol. Biosci., vol. 8, no. 8, pp. 758-765, Aug. 2008.

[18] Y. B. Choy, H. Choi, and K. Kim, "Uniform biodegradable hydrogel microspheres fabricated by a surfactant-free electric-field-assisted method," Macromol. Biosci., vol. 7, no. 4, pp. 423-428, Apr. 2007.

[19] G. Hermanson, "EDC plus sulfo-NHS," in Bioconjugate Techniques, 2nd ed. New York: Academic, 2008, pp. 216-220.

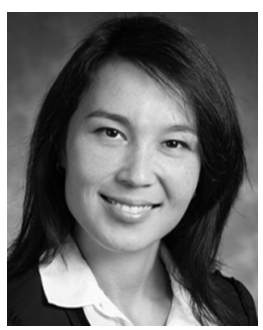

Gulsim K. Kulsharova received the B.S. in engineering physics and M.S. in electrical engineering degrees from University of Illinois at Urbana-Champaign, IL, USA, in 2010 and 2012, respectively. Her undergraduate and graduate works focused on microfluidic compact disk development for lab-on-chip applications and nanoparticle based targeted drug delivery for cancer treatment. Her current research interests are also in biomaterials, noble metal nanoparticles for therapeutic applications.

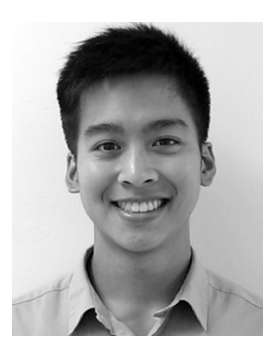

Matthew B. Lee received the B.S. and M.S. degrees in molecular and cell biology from the University of Illinois, Urbana-Champaign, IL, USA, in 2010 and 2012 respectively. His graduate work focused on ultrasound characterization of nanoparticles and their application in localized drug delivery.

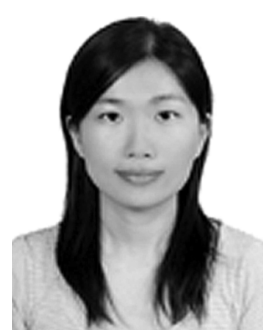

Felice Cheng received her Ph.D degree in electrical and computer engineering from University of Illinois at Champaign-Urbana, IL, USA, in 2011. Later, she worked as postdoctoral fellow in Thin Film \& Charged Particle Research Laboratory, University of Illinois at Champaign-Urbana. In 2012, she started her research career in Pharmaceutical Optimization Technology Division, Biomedical Technology and Device Research Laboratories, Industrial Technology Research Institute, and is responsible fo the formulation research area, especially for the polymeric microparticles and hydrogel.

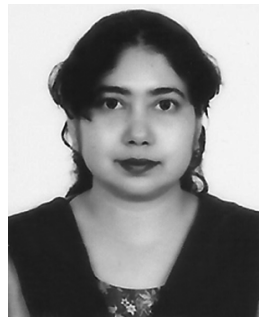

Munima Haque received her M.S. from Southern Illinois University, Carbondale, IL, USA, and her $\mathrm{Ph} . \mathrm{D}$. from University of Illinois Urbana-Champaign, IL, USA (UIUC). She worked as a Postdoctoral Research Associate at Micro and Nanotechnology Laboratory ECE UIUC, and her work involves targeted drug delivery for cancer cells, gene therapy. Her research interests also include radiation health effects in living systems and environments, various factors affecting cancer occurrence and reoccurrence.

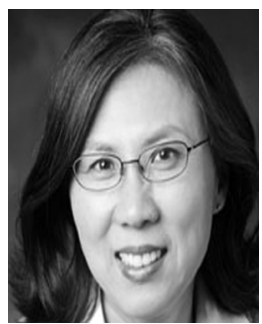

Hyungsoo Choi received the B.S. and Ph. D. degrees from Seoul National University, Korea, and Brown University, Providence, RI, USA, respectively. After postdoctoral training at the University of Michigan, Ann Arbor, MI, USA, she has worked for the Korea Institute of Science and Technology and the University of Illinois at Urbana-Champaign. Her research interests include micro and nanosphere mediated advanced drug delivery as well as the synthesis and development of tailored organometallic, inorganic and polymeric precursors to produce nanostructured materials, such as thin films, nanoparticles, and nanowires of metals and ceramics.

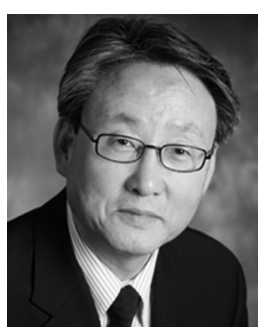

Kyekyoon Kim received the B.S. degree in nuclear engineering from Seoul National University, Korea, and both the M.S. and Ph.D. degree from Cornell University, Ithaca, NY, USA. He is Professor of Electrical and Computer Engineering, Bioengineering, Neuroscience Program, Materials Science and Engineering, and Nuclear, Plasma, and Radiological Engineering, and Director of Thin Film and Charged Particle Research Laboratory, at the University of Illinois at Urbana-Champaign IL, USA. His research has included GaN-based power electronic devices, electrostatic spraying, precision particles, controlled release, biotechnology, nanotechnology, solar cells, thin films, controlled thermonuclear fusion, and plasma physics.

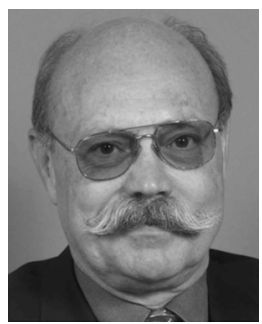

William D. O'Brien, Jr. (S'64-M'70-SM'79F'89-LF'08) received the B.S., M.S., and Ph.D. degrees from the University of Illinois, Urbana-Champaign, IL, USA. Since 1971 he has worked for FDA and UIUC. His research interests involve the many areas of ultrasound-tissue interaction, including biological effects and quantitative ultrasound imaging for which he has published 386 papers.

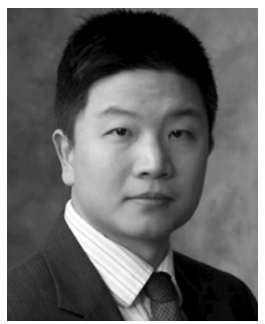

Dr. Logan Liu received his Ph.D. degree in bioengineering from University of California (UC)-Berkeley, CA, USA, and UC-San Francisco, CA, USA. He finished his postdoctoral training in the Helen Diller comprehensive cancer center at San Francisco as well as Lawrence Livermore National Lab where he was a Lawrence fellow. In 2008 he joined University of Illinois at Urbana-Champaign, IL, USA, as an Assistant Professor in Department of Electrical and Computer Engineering and Department of Bioengineering. $\mathrm{He}$ is also a faculty fellow of National Center for Supercomputing Applications (NCSA). He has published over 80 journal and conference papers as well as several book chapters. His research focuses on designing and developing integrative bionano and microfluidic technologies and devices for the applications of advanced nanomanufacturing, bioimaging, cancer diagnostics and therapy, environmental sensing and mobile health. 